PO-0575 INDICATIONS AND OUTCOMES OF LUMBAR PUNCTURES IN PRETERM NEONATES IN A TERTIARY NEONATAL UNIT

H Wood, E Seager, J Gray, SV Rasiah. Neonatology, Birmingham Women's NHS Foundation Trust, Birmingham, UK

\subsection{6/archdischild-2014-307384.1216}

Background Lumbar puncture (LP) is usually performed when there is a clinical suspicion of meningitis in babies with suspected sepsis. Meningitis can be challenging to diagnose in preterm babies.

Aim To audit the indications and outcomes of LPs performed in preterm babies $(<37+0$ weeks $)$ in a tertiary neonatal unit.

Methods A list of preterm babies who had an LP was obtained from the Microbiology Department between 01/01/2010 and $31 / 12 / 2013$. The Badger electronic patient record and hospital blood results systems were reviewed to collect the data.

Results In the last 4 years we had 2618 preterm babies admitted to the neonatal unit. 98 LPs were performed in 89 preterm babies. The reasons for LPs were; (a) raised CRP in 60 cases (median CRP was 65), (b) positive blood culture in 28 cases, (c) abnormal neurology in 13 cases, (d) other reasons in 13 cases. In some cases, an LP was indicated by a combination of these factors. There were two positive cultures; one with Group B Streptococcus and another with Serratia Marcescens. At discharge, 5 had a diagnosis of meningitis based on microscopy and/or culture.

Conclusion The predominant indication for LPs in preterm babies was a raised CRP followed by a positive blood culture. We only isolated organisms from two samples. Diagnosing meningitis in preterm babies remains challenging but should always be suspected in the presence of a raised CRP and positive blood culture.

\section{PO-0576 DIFFERENTIAL LIPOPOLYSACCHARIDE-INDUCED MIRNA EXPRESSION PROFILE AND TLR SIGNALLING GENES IN LEUKOCYTES FROM NEWBORNS AND ADULTS}

Y Yang, C Chen. Pediatrics Institute, Children's Hospital of Fudan University, Shanghai, China

\subsection{6/archdischild-2014-307384.1217}

Background and aims Newborns are more susceptible to microbial infections than are adults. To explore the molecular mechanisms underlying the increased susceptibility of newborns to infection, we compared the expression profile of miRNAs in response to LPS stimulation in vitro in leukocytes from newborns and adults.

Methods We obtained blood samples from 10 healthy term newborns and 11 healthy adults. Whole blood cells were cultured in vitro with or without LPS. After $2 \mathrm{~h}$ of culture, leukocytes were isolated, and total RNA was extracted. The miRCURY LNA Array (v.14.0) was used to detect miRNAs expression profile. TLR-related genes were studied by microarray and PCR arrays. A bioinformatics analysis was used to identify the potential biological processes and targets involved in the TLR signals affected by these miRNAs.

Results A total of 53 miRNAs and 29 TLR-related genes were differentially expressed between newborns and adults. The bioinformatics analysis showed that the potential target genes of these differential miRNAs were involved in regulation of cellular biosynthetic process, regulation of gene expression, regulation of macromolecule biosynthetic process, etc. Twelve potential miRNA-mRNA interaction sites were found within the cDNA sequences of ten differentially expressed TLR signalling pathway genes.

Conclusions We identified a differential miRNA expression profile during LPS-induced acute inflammation in leukocytes derived from newborns and adults. The target genes of these differential miRNA were mainly involved in several biological processes, and these miRNAs may play important roles in the regulation of TLR signals. However, the precise mechanisms require further validation.

\section{PO-0577 "OLD" AND "NEW" MARKERS IN EARLY NEONATAL SEPSIS - DIAGNOSIS VALUE}

${ }^{1} \mathrm{G}$ Zaharie, ${ }^{1} \mathrm{M}$ Hasmasanu, ${ }^{1} \mathrm{~L}$ Blaga, ${ }^{2} \mathrm{~T}$ Zaharie, ${ }^{1} \mathrm{M}$ Matyas. ${ }^{1}$ Neonatology, University of Medicine and Pharmacy Iuliu Hatieganu CLUJ-NAPOCA, CLUJ-NAPOCA, Romania; ${ }^{2}$ Pathology, Regional Institut of Gastro-Enterology and Hepathology, CLUJ-NAPOCA, Romania

\subsection{6/archdischild-2014-307384.1218}

Background and aim To evaluate the diagnosis value of TLR-2 (Toll-like Receptors), TLR-4, IL-6 (interleukine -6), TNF- $\alpha$ (tumour necrosis factor $-\alpha$ ) and $\mathrm{CRP}(\mathrm{C}$ reactive protein) in the diagnosis of early neonatal sepsis at the premature babies with premature rupture of the membranes.

Material and methods Diagnosis of sepsis was done according with International Sepsis Definitions Criteria. Study group involved newborns with signs and symptoms suggestive for systemic infection, requiring full sepsis evaluation and antibiotic treatment and control group is represented by healthy newborns. We determined in the I-st day TLR-2, TLR-4, IL-6, TNF- $\alpha$ and CRP and in the III-th day the same without TLR. We used latex agglutination test for CRP, Elisa technique for TNF- $\alpha$ and IL-6 and flow cytometry for TLR. Statistical analysis was done with "Statistica VI".

Results Sepsis group presented in the I-st day: TNF- $\alpha(\mathrm{pg} / \mathrm{ml})=$ $14,7[5,0-24,3]$; IL6 $(\mathrm{pg} / \mathrm{ml})=153,7[82,3-225,1]$; CRP $(\mathrm{mg} \%)=$ $0,83[0,54-1,12]$; and the expression (\%) of TLR2 $=42,5$ $[29,5-55,4]$; and TLR4 $=2,2[1,26-3,15]$. TNF- $\alpha$ correlates significantly and negative with TLR2. TLR2 correlates significantly and positive with TLR4. In the 3-rd day: TNF- $\alpha(\mathrm{pg} / \mathrm{ml})=10,1$ $[5,1-15,1] ;$ IL6 $(\mathrm{pg} / \mathrm{ml})=46,5[16,3-76,7]$; CRP $(\mathrm{mg} \%)=1,2[0,6-$ 1,81]. Control group presented: TLR2 $(\%)=5,69(\mathrm{p}=$ $0,00006)$ and $\operatorname{TLR} 4(\%)=0,67(\mathrm{p}=0,037)$. In the first day TNF- $\alpha$ and IL6 were higher in study group vs. control group but no statistical differences.

Conclusions TLR-2 and TLR-4 could confirm like markers the neonatal sepsis.

IL- 6 and TNF- $\alpha$ consider to be markers of early neonatal sepsis.

CRP could not be consider like marker for early neonatal sepsis.

P0-0578 PHOTOTHERAPY DECREASES THE SERUM GLOBULIN CONCENTRATION IN NEWBORN HYPERBILIRUBINEMIA?

J Zheng, D Zhao. Pediatrics, Zhongnan Hospital of Wuhan University, Wuhan, China

10.1136/archdischild-2014-307384.1219 


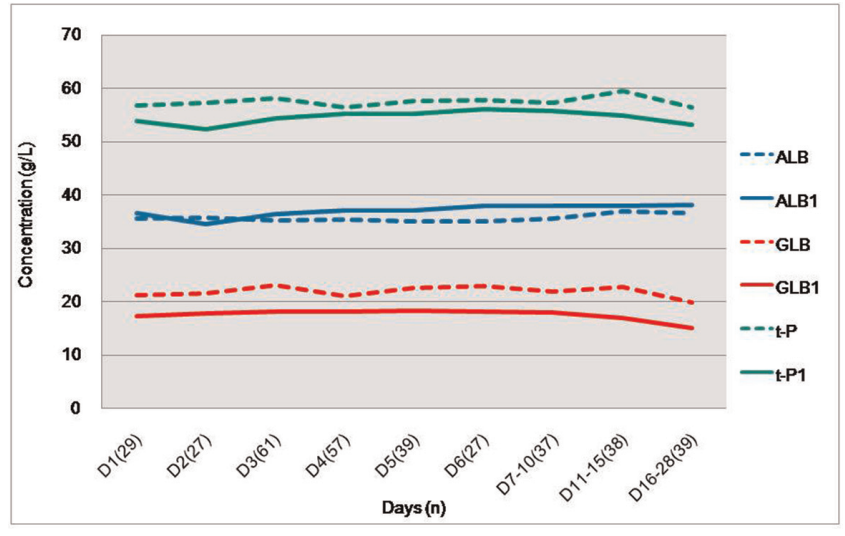

\section{Abstract P0-0578 Figure 1}

Objective To assess the impact of phototherapy as the risk factor on the reduction of serum globulin in neonatal hyperbilirubinemia.

Methods Total of 430 full term infants aged at 1-28 days diagnosed with neonatal hyperbilirubinemia was enrolled in this study. Intrauterine infection, genetic abnormal and congenital diseases was excluded. All newborns received single-side phototherapy (halogen lamps for $12 \mathrm{~h}$ per day, 3 days) or plus intravenous albumin (1 g/kg.d, two days) or plus intravenous immuneglobulin ( $1 \mathrm{~g} / \mathrm{kg} . \mathrm{d}$, two days). The total serum bilirubin (TSB), albumin (ALB) and globulin (GLB) levels were detected twice at the first day and fourth day of hospitalisation respectively.

Results TSB concentrations decreased from $299.6 \pm 83.9 \mu \mathrm{mol}$ to $163.6 \pm 57.6 \mu \mathrm{mol} / \mathrm{L}$ after three days intensive treatment $(\mathrm{p}$ $<0.001)$. Pearson correlative analysis shows that TSB is significant correlated to GLB level $(r=0.245, p<0.01)$ and not related to ALB. There was a significant reduction of GLB levels in each age groups after treatment $(\mathrm{p}<0.001)$. The GLB concentrations decreased 2-4 g/L (10-20\% compared to their basic levels) and dramatically decreases in groups of $>7$ days of birth age $(\mathrm{p}<0.001)$. The reductions of GLB level were from $21.3 \pm$ $4.1 \mathrm{~g} / \mathrm{L}$ to $18.5 \pm 4.2 \mathrm{~g} / \mathrm{L}$ in phototherapy group, and $23.0 \pm$ $3.9 \mathrm{~g} / \mathrm{L}$ to $16.6 \pm 4.5 \mathrm{~g} / \mathrm{L}$ in phototherapy plus IVALB $(\mathrm{p}<$ 0.001). The effect of phototherapy on reduction of GLB levels was correlated to the ages of birth.

Conclusions These results demonstrated that phototherapy accelerates serum globulin clearance which implies infants facing to the risk of immunity injure, especially in age over 16 days, while additional IVALB aggravated the reduction along with the ages.

\section{Neonatal Infections}

\section{PO-0578a EPIDEMIOLOGY AND ANTIBIOTIC SUSCEPTIBILITY OF GRAM-NEGATIVE (GN) NEONATAL INFECTIONS OVER 10 YEARS: DATA FROM THE NEONIN INFECTION SURVEILLANCE NETWORK (WWW.NEONIN.ORG.UK)}

${ }^{1} \mathrm{C}$ Kortsalioudaki, ${ }^{1} \mathrm{~A}$ Kent, ${ }^{2} \mathrm{~N}$ Kennea, ${ }^{3} \mathrm{P}$ Clarke, ${ }^{4} \mathrm{~T}$ Watts, ${ }^{5} \mathrm{~N}$ Embleton, ${ }^{6} \mathrm{P}$ Satodia, ${ }^{7} \mathrm{~T}^{1}$ Scorrer, ${ }^{8} \mathrm{~J}$ Chang, ${ }^{9} \mathrm{R}$ Geethanath, ${ }^{1} \mathrm{PT}$ Heath. ${ }^{1}$ Paediatric Infectious Diseases Research Group Clinical Sciences, St George's University of London, London, UK; '²Neonatal Services, St George's Healthcare Trust, London, UK; ${ }^{3}$ Neonatal Services, Norfolk and Norwich University Hospital, Norwich, UK; ${ }^{4}$ Neonatal Services, Evelina London Children's Hospital Guy's and St Thomas' NHS Foundation Trust, London, UK; ${ }^{5}$ Newcastle Neonatal Services, Royal Victoria Infirmary Newcastle Upon Tyne, Newcastle, UK; ${ }^{6}$ Neonatal Services, University Hospital of Coventry and Warwickshire, Coventry, UK; ${ }^{7}$ Neonatal
Services, Queen Alexandra Hospital, Portsmouth, UK; ${ }^{8}$ Neonatal Services, Croydon University Hospital, London, UK; ${ }^{9}$ Neonatal Services, City Hospitals Sunderland (Sunderland Royal Hospital), Sunderland Tyne and Wear, UK

\subsection{6/archdischild-2014-307384.1220}

Background and aims Gram-negative sepsis is associated with high morbidity and mortality in neonates and necessitates prompt treatment with appropriate antibiotics. This study focused on the epidemiology and antibiotic susceptibility of GN pathogens over the last 10 years using data from a neonatal infection network.

Methods neonIN is an international web-based surveillance database which captures culture proven neonatal infections. Data for UK neonatal-units (NNUs) on GN infection episodes between April 2004 and May 2014 were extracted. Late-onset sepsis (LOS) was defined as an episode occurring from 48-hours after birth.

Results There were 605 episodes from 28 NNUs (involving 540 neonates). Overall incidence was $0.87 / 1000$ live-births and 7.10 / 1000 NNU-admissions. LOS accounted for the majority of all GN episodes $(532,87.9 \%)$ and was associated with an earlier gestation-age than early-onset sepsis (median 26 vs 30 weeks, p $<0.001)$. E. coli was the commonest pathogen $(217,35.9 \%)$ followed by Klebsiella sp. (120, 19.8\%) and Enterobacter sp. (102, $16.9 \%)$. The pathogens were predominately isolated from blood (544, 89.9\%). 74 (12.2\%) episodes were treated as meningitis with no significant difference in meningitis rates between pathogens. Resistance data were available for 342 (56.5\%) episodes. Resistance to 3rd-generation cephalosporins was $19.7 \%$ (36/ 183), to aminoglycosides $9.9 \%(29 / 291)$ and to quinolones $13.1 \%(23 / 175)$.

Conclusion GN infections represent a significant burden of infection in the hospitalised neonate. Rates of 3rd-generation cephalosporin resistance pose a challenge for their use as empiric therapy. Ongoing surveillance of antibiotic susceptibility is necessary to ensure optimal antibiotic practice.

On behalf of the Neonatal Infection Surveillance Network (neonIN).

\section{Neonatal Nutrition and Gastroenterology}

\section{PO-0579 RISK FACTORS FOR IRON DEFICIENCY AND IRON DEFICIENCY ANAEMIA IN LATE PRETERM INFANTS AT THE AGE OF 6 WEEKS}

${ }^{1}$ MD Akkermans, ${ }^{1} \mathrm{~L}$ Uijterschout, ${ }^{2} \mathrm{JB}$ van Goudoever, ${ }^{1} \mathrm{~F}$ Brus. ${ }^{1}$ Pediatrics, Juliana Children's Hospital/Haga Hospital, The Hague, Netherlands; ${ }^{2}$ Pediatrics, Academic Medical Center Amsterdam and VU University Medical Center Amsterdam, Amsterdam, Netherlands

\subsection{6/archdischild-2014-307384.1221}

Background and aims Iron deficiency (ID) has long-term detrimental effects on neurodevelopment. Preterm infants are at risk for developing ID or iron deficiency anaemia (IDA) during the first weeks of life. The aim of this study was to identify early risk factors during hospitalisation for a deprived iron status in late preterm infants at the age of 6 weeks.

Methods We analysed the iron status of 99 infants born between 32 and 35 weeks of gestational age from March 2011 to May 2013 in three non-tertiary hospitals in the Netherlands. ID and IDA at the age of 6 weeks were defined as a ferritin concentration $<70 \mu \mathrm{g} / \mathrm{L}$ and the combination of a haemoglobin level $<$ $110 \mathrm{mg} / \mathrm{dL}$ and ID, respectively. 